EPJ Web of Conferences 60, 17004 (2013)

DOI: $10.1051 /$ epjconf $/ 20136017004$

(C) Owned by the authors, published by EDP Sciences, 2013

\title{
Searches for monojets and monophotons with the ATLAS detector
}

\author{
Jalal Abdallah ${ }^{1, a}$, on behalf of the ATLAS Collaboration \\ ${ }^{1}$ Institut de Física d'Altes Energies (IFAE) \\ Edifici Cn, \\ Universitat Autònoma de Barcelona (UAB), \\ E-08193 Bellaterra (Barcelona), Spain
}

\begin{abstract}
.
Studies of events with a single high energy jet or photon, and large missing transverse momentum at the Large Hadron Collider is one of the most effective ways to search for physics beyond the Standard Model. The background from the Standard Model processes is dominated by jet/photon production associated with Z or W bosons undergoing leptonic decays to neutrinos and/or unidentified electrons or muons. The sensitivity to new physics signals relies on the good understanding and the accurate estimation of these backgrounds, and therefore the use of data-driven techniques is often required. In this article, I will present a compilation of the latest results from the monojet and monophoton searches based on the analysis of the 2011 and 2012 proton-proton collision data collected with the ATLAS detector at a center-of-mass energy of $7 \mathrm{TeV}$ and $8 \mathrm{TeV}$, respectively. The results are interpreted in the context of various models beyond the Standard Model including large extra dimensions, gauge mediated supersymmetry breaking and effective field theory of dark matter production.
\end{abstract}

\section{Introduction}

Events with an energetic jet or photon, and large missing momentum in the final state are a distinctive signature in searches for new physics at colliders. In particular, monojet and monophoton events have been considered to search for supersymmetry, large extra dimensions (LED) and weakly interacting massive particles (WIMPs) as candidates for dark matter.

The LED model of Arkani-Hamed, Dimopoulos, and Dvali (ADD) [1] proposes an explanation for the large difference between the electroweak unification scale $O\left(10^{2}\right) \mathrm{GeV}$ and the Planck scale $M_{P l} \sim O\left(10^{19}\right) \mathrm{GeV}$ by postulating the presence of $n$ extra spatial dimensions of size $R$, and redefining the Planck scale in $4+n$ dimensions. The higher dimensional Planck scale $M_{D}$ is given by $M_{P l}{ }^{2} \sim M_{D}{ }^{2+n} R^{n}$. For an appropriate choice of $R$ for a given $n^{1}$ on could obtain values for $M_{D}$ close to the electroweak scale. The compactification of the extra spatial dimensions leads to a Kaluza-Klein tower of massive graviton modes. At hadron colliders these gravitons, which can be produced in association with a jet/photon, escape detection into the extra dimensions leading to monojet or monophoton final states

Evidence of the existence of non-baryonic dark matter in the universe is concluded from many cosmological observations, including the anomaly in the rotational velocities of galaxies and clusters of galaxies, the lensing

\footnotetext{
a e-mail: jalal.abdallah@ cern.ch

${ }^{1}$ The case of $n=1$ is excluded due to a significant modification of the Newtonian law that would be visible at the scale of the solar system.
}

effects of distant galaxies, etc. A weakly interacting massive particle WIMP $\chi$ with mass $m_{\chi}$ in the range between $1 \mathrm{GeV}$ and a few $\mathrm{TeV}$ constitutes a possible explanation for dark matter. In direct detection experiments, the scattering of WIMP particles on heavy nuclei is searched for by measuring ionization, scintillation and phonon signals. The detection of energetic cosmic rays constitutes an indirect way to search for dark matter from $\chi \bar{\chi}$ annihilation. At colliders WIMP particles could be pair-produced, and detected in the case where an initial jet or photon has been emitted. The interaction of WIMPs with Standard Model (SM) particles is studied using different effective field theory operators [2] where the mediator of the interaction is assumed to be at the $\mathrm{TeV}$ scale. The vertex coupling is suppressed by an effective cut-off mass scale $M_{*}$.

In gauge-mediated SUSY breaking (GMSB) scenarios [3], the gravitino $\tilde{G}$ (spin- $\frac{3}{2}$ superpartner of the graviton) is often considered the lightest supersymmetric particle (LSP) and a potential candidate for dark matter, where its mass is related to the SUSY breaking scale $F$ and $M_{P l}$ via $m_{\tilde{G}} \propto F / M_{P l}$.

At hadron colliders the cross sections for associate production of gravitino-squark $(p p \rightarrow \tilde{G} \tilde{q}+X)$ and gravitinogluino $(p p \rightarrow \tilde{G} \tilde{g}+X)$ processes are sizable if the gravitino is light enough. The decay of the gluino or squark into a gravitino and a gluon $(\tilde{g} \rightarrow \tilde{G} g)$ or a gravitino and a quark ( $\tilde{q} \rightarrow \tilde{G} q)$, leads to final states with a pair of gravitinos that escape detection and an energetic jet.

In this article, we present a compilation of the results of the search for new phenomena in monojet and 
monophoton final states with the ATLAS detector [4] based on $7 \mathrm{TeV}$ proton-proton collision data corresponding to $4.7 \mathrm{fb}^{-1}$ of integrated luminosity and updated to $10.5 \mathrm{fb}^{-1}$ of $8 \mathrm{TeV}$ data. All the results presented in this article can be found with more details in Ref. [5-7]. For the specific definition of the reconstructed physics objects, the reader is redirected to the above references.

\subsection{Event selection}

The data considered in the analyses have been collected with the ATLAS detector fully operational.

\section{Preselection}

The preselection of events is very similar in both of the monojet and the monophoton analyses. The events are selected using inclusive missing transverse momentum $\left(E_{\mathrm{T}}^{\text {miss }}\right)$ triggers which are more than $95 \%$ efficient for the $E_{\mathrm{T}}^{\text {miss }}$ offline requirements. The events are further required to have a reconstructed primary vertex in order to reject beam-related backgrounds and cosmic rays. Also, the events are rejected if they present a jet with anomalous charge fraction of electromagnetic fraction (fraction of transverse momentum associated with the tracks or measured in the electromagnetic calorimeter). Additional requirements are applied to suppress coherent noise and electronic noise bursts in the calorimeter producing anomalous energy deposits. Events are required to have no identified electrons or muons.

\section{Monojet signal region selection}

Events are required to have $E_{\mathrm{T}}^{\mathrm{miss}}>120 \mathrm{GeV}$, a jet with $p_{\mathrm{T}}>120 \mathrm{GeV}$ and $\left|\eta^{\text {jet }}\right|<2$, and at most two jets with the second leading $p_{\mathrm{T}}$ above $30 \mathrm{GeV}$ in the region $|\eta|<4.5$. For the events with secondary jet, an azimuthal separation $\Delta \phi\left(\right.$ jet, $\left.E_{\mathrm{T}}^{\text {miss }}\right)>0.5$ between the $E_{\mathrm{T}}^{\text {miss }}$ and the direction of the second leading jet is required. This requirement reduces the multijet background contribution where the large $E_{\mathrm{T}}^{\mathrm{miss}}$ originates from the mis-measurement of the secondleading jet $p_{\mathrm{T}}$. Four inclusive signal regions denoted SR1 to SR4 are defined with symmetric lower thresholds on the leading jet $p_{\mathrm{T}}$ and $E_{\mathrm{T}}^{\mathrm{miss}}$, with values of $120,220,350$ and $500 \mathrm{GeV}$.

\section{Monophoton signal region selection}

Events are required to have $E_{\mathrm{T}}^{\text {miss }}>150 \mathrm{GeV}$ and a photon with $p_{\mathrm{T}}>150 \mathrm{GeV}$ in $\left|\eta^{\text {jet }}\right|<2.37$, and at most one jet with $p_{\mathrm{T}}$ above $30 \mathrm{GeV}$ in the region $|\eta|<4.5$. Similarly to the monojet selection, azimuthal separation $\Delta \phi\left(\gamma, E_{\mathrm{T}}^{\mathrm{miss}}\right)>$ $0.4, \Delta \phi\left(\right.$ jet, $\left.E_{\mathrm{T}}^{\mathrm{miss}}\right)>0.4$ and $\Delta \phi(\gamma$, jet $)>0.4$ are imposed.

\subsection{Background estimation}

The dominant processes contributing to the background are $Z / W+$ jets and $Z / W+\gamma$, where the $Z$ and the $W$ decay into neutrinos and/or unidentified leptons. For the monophoton selection an additional contribution from $Z / W+$ jets arises from an electron or a jet mis-identified as a photon. The remaining sub-dominant backgrounds consist of processes involving the top quark and diboson production. The multijet $/ \gamma+$ jet processes and the noncollision background from beam halo and cosmic rays are estimated from data. Due to the large uncertainty on their estimation, their contributions to the total background are maintained at the percent level by the dedicated cuts described above.

Data-driven methods are employed in both of the monojet and the monophoton analyses in order to estimate the different electroweak background contributions. Control samples in data, orthogonal to the signal regions, with identified electrons or muons in the final state, with the same $E_{\mathrm{T}}^{\mathrm{miss}}$ and jet requirements as in the signal regions are used to determine the $W / Z+$ jets and $W / Z+\gamma$ backgrounds. This reduces significantly the relatively large theoretical and experimental systematic uncertainties associated with purely MC-based predictions. In order to emulate the $W / Z+$ jets background in the signal regions, the leptons in the control regions are treated as neutrinos in the computation of the $E_{\mathrm{T}}^{\mathrm{miss}}$. For the monophoton analysis, due to limited statistics, an inclusive one muon control region is considered. In the monojet analysis, the following control regions are considered ${ }^{2}$.

The $W(\rightarrow \mu v)+$ jets control sample is defined using events with a muon with $p_{\mathrm{T}}>7 \mathrm{GeV}$ and transverse mass ${ }^{3}$ in the region $40 \mathrm{GeV}<m_{\mathrm{T}}<100 \mathrm{GeV}$. Similarly, a $Z / \gamma^{*}\left(\rightarrow \mu^{+} \mu^{-}\right)+$jets control sample is selected requiring the presence of two muons with invariant mass in the range $76 \mathrm{GeV}<m_{\mu \mu}<116 \mathrm{GeV}$. Finally, a $W(\rightarrow e v)+$ jets dominated control sample is defined using events with an electron candidate with $p_{\mathrm{T}}>20 \mathrm{GeV}$.

Figure 1 shows the distribution of the transverse mass in the muon control region while figure 2 shows the distribution of $E_{\mathrm{T}}^{\text {miss }}$ in the $W(\rightarrow \mu v)$ control region for SR1 kinematics of the monojet selection. The $W / Z+$ jets predictions are obtained using ALPGEN [8]. The ratio data to Monte Carlo (MC) simulation is also shown.

As an example of the background estimation in the case of the dominant $\mathrm{Z}(\rightarrow v \bar{v})+$ jets process, its contribution to a signal region in a given bin of a given distribution $N(\mathrm{Z}(\rightarrow v \bar{v})+\text { jets })_{\text {signal }}$ would be determined using the $W(\rightarrow \mu v)+$ jets control sample in data according to ${ }^{4}$

$$
\begin{aligned}
& N(\mathrm{Z}(\rightarrow v \bar{v})+\text { jets })_{\text {signal }}=\left(N_{W \rightarrow \mu v, \text { control }}^{\text {data }}-N_{W, \text { control }}^{\text {backround }}\right) \\
& \times \frac{N^{M C}(\mathrm{Z}(\rightarrow v \bar{v})+\text { jets })_{\text {signal }}}{N_{W \rightarrow \mu \nu, \text { control }}^{M C}}
\end{aligned}
$$

Different sources of uncertainties are considered including jet and $E_{\mathrm{T}}^{\text {miss }}$ energy scale and resolution, lepton identification efficiencies, normalization of the top quark

\footnotetext{
${ }^{2}$ In the $7 \mathrm{TeV}$ monojet analysis [6], the definition of the control regions is slightly different from the text.

${ }^{3}$ The transverse mass $m_{\mathrm{T}}$ is defined by the lepton and neutrino $p_{\mathrm{T}}$ and $\phi$ as $m_{T}=\sqrt{2 p_{\mathrm{T}}^{\ell} p_{\mathrm{T}}^{v}\left(1-\cos \left(\phi^{\ell}-\phi^{v}\right)\right)}$.

${ }^{4}$ In the case of the monophoton analysis, the formula is applied on the total number of events and not on each bin of the distribution.
} 


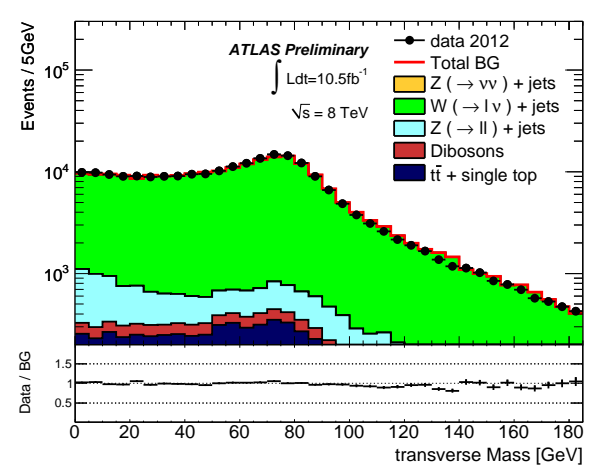

Figure 1: Transverse mass distribution in the muon control region, for the SR1 selection, compared to the background predictions [5].

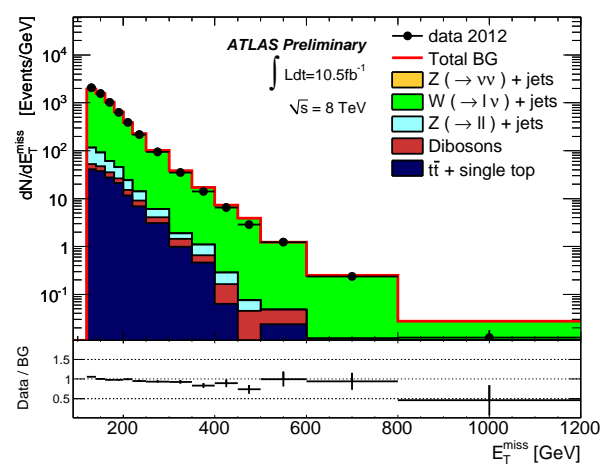

Figure 2: $E_{\mathrm{T}}^{\mathrm{miss}}$ distribution in the $W(\rightarrow \mu v)+$ jets control region, for the SR1 selection, compared to the background predictions [5].

and diboson backgrounds and also the statistical uncertainties of the measured data distributions in the control regions, as well as the statistical uncertainties on the MC. Modeling uncertainties on the parton shower, parton distribution functions (PDFs), renormalization and factorization scales are also evaluated and generally found to be at the level of $3 \%$ to $6 \%$. In the monojet analysis, this uncertainty dominates for the low $p_{\mathrm{T}}$ signal regions SR1, 2 while for high $p_{\mathrm{T}}$ signal regions the statistical uncertainties from data in the control region and $\mathrm{MC}$ (up to $15 \%$ ) become more relevant. In the monophoton analysis, the total uncertainty on the background estimation is $13 \%$ and is dominated by the statistical uncertainty on data in the muon control region.

\subsection{Results and interpretation}

Good agreement is observed between the data and the SM predictions in both of the monojet and monophoton analyses. For the $7 \mathrm{TeV}$ monojet analysis [6] SR4 is used to set limits while for the $8 \mathrm{TeV}$ analysis [5] it is SR3 that is

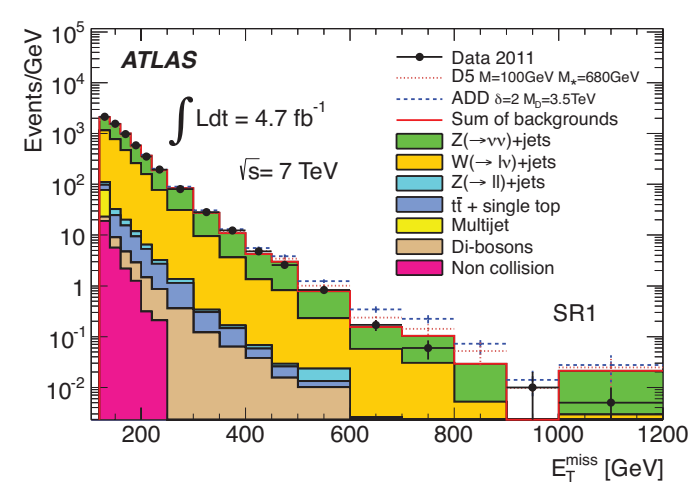

Figure 3: Measured $E_{\mathrm{T}}^{\text {miss }}$ distribution compared to the predictions for SM backgrounds in SR1 at $7 \mathrm{TeV}$ [6].

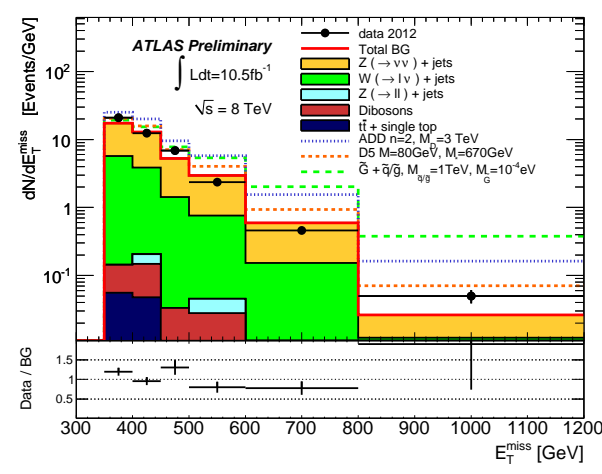

Figure 4: Measured $E_{\mathrm{T}}^{\text {miss }}$ distribution (black dots) in SR3 compared to the predictions for SM backgrounds at $8 \mathrm{TeV}$. Only statistical uncertainties are shown. For illustration purposes, the impact of different ADD, WIMP, and GMSB scenarios are included [5].

used because SR4 was suffering from a sizable statistical uncertainties from the Monte Carlo simulation.

Figures 3 and 4 show the measured $E_{\mathrm{T}}^{\text {miss }}$ distributions in SR1 at $7 \mathrm{TeV}$ and in SR3 at $8 \mathrm{TeV}$, respectively, for the monojet analysis. Figures 5 and 6 show the measured $E_{\mathrm{T}}^{\text {miss }}$ and leading photon $p_{\mathrm{T}}$ distributions for the monophoton analysis at $7 \mathrm{TeV}$. The impact of different ADD, WIMP, and GMSB signals is stacked over the total background predictions.

\subsubsection{Large Extra Dimensions}

The results of the monojet and the monophoton analyses allow to independently set limits on the parameters of the ADD model.

Different sources of systematic uncertainties including jet and $E_{\mathrm{T}}^{\mathrm{miss}}$ scales and resolutions, trigger efficiency and luminosity are considered on the ADD prediction. The total experimental uncertainty is generally at the level of $10 \%$. Uncertainties on the modeling of the initial- and final-state radiation (ISR and FSR), PDFs and renormal- 


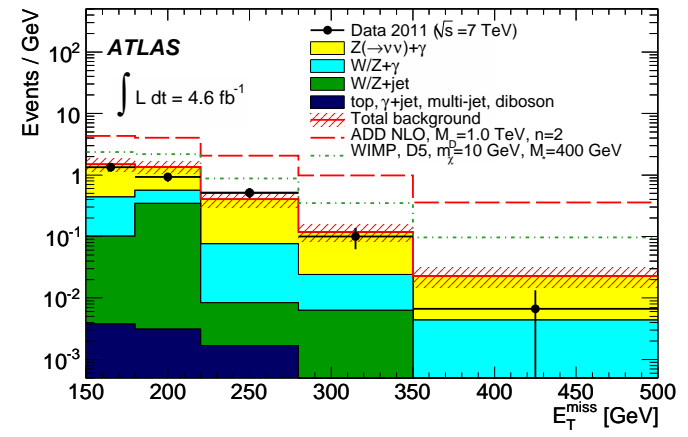

Figure 5: The measured $E_{\mathrm{T}}^{\text {miss }}$ distribution (black dots) compared to the SM (solid lines), SM+ADD LED (dashed lines), and SM+WIMP (dotted lines) predictions, for two particular ADD LED and WIMP scenarios in the monophoton analysis. The band around the total background prediction includes uncertainties on the datadriven background estimates and statistical uncertainties on MC samples [7].

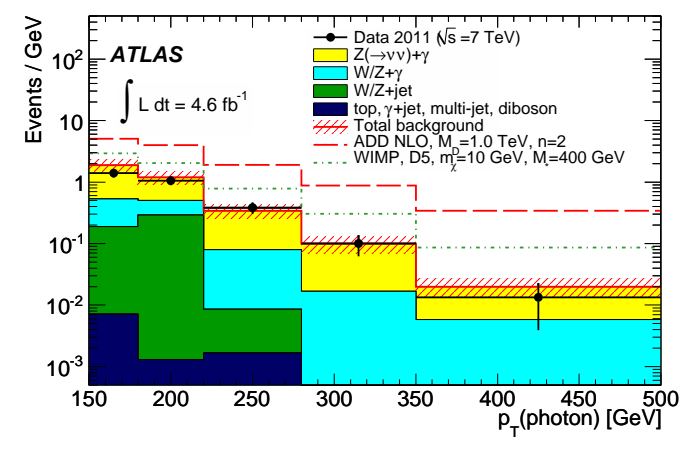

Figure 6: The measured photon $p_{\mathrm{T}}$ distribution (black dots) compared to the SM (solid lines), SM+ADD LED (dashed lines), and SM+WIMP (dotted lines) predictions, for two particular ADD LED and WIMP scenarios in the monophoton analysis. The band around the total background prediction includes uncertainties on the datadriven background estimates and statistical uncertainties on MC samples [7].

ization and factorization scales are determined using simulated data. They result in total uncertainties between $20 \%$ and $50 \%$ on the predicted signal yields.

Figures 7 and 8 show the expected and observed $95 \%$ CL lower limits on $M_{D}$ as a function of the number of extra dimensions considered in the ADD model for the monojet and monophoton analyses, respectively. They are both based on the results of the analyses of the $7 \mathrm{TeV}$ data with $4.7 \mathrm{fb}^{-1}$. The difference in the exclusion pattern between the two analysis over the number of extra dimensions is due to the fact that the cross sections of ADD signals are very close around $M_{D} \sim 2 \mathrm{TeV}$ while at larger $M_{D}$ values the cross sections are higher for lower number of extra dimensions. The $8 \mathrm{TeV}$ monojet results with $10.5 \mathrm{fb}^{-1}$ do

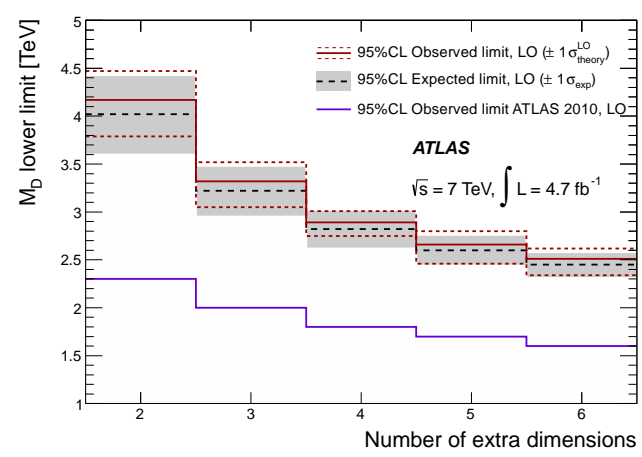

Figure 7: 90\% CL lower limits on $M_{D}$ for different numbers of extra dimensions based on SR4. Observed and expected limits including all but the theoretical signal uncertainties are shown as solid and dashed lines, respectively. The grey $\pm 1 \sigma$ band around the expected limit is the variation expected from statistical fluctuations and experimental systematic uncertainties on the SM and signal processes. The impact of the theoretical uncertainties is shown by the red small-dashed $\pm 1 \sigma$ limit[6].

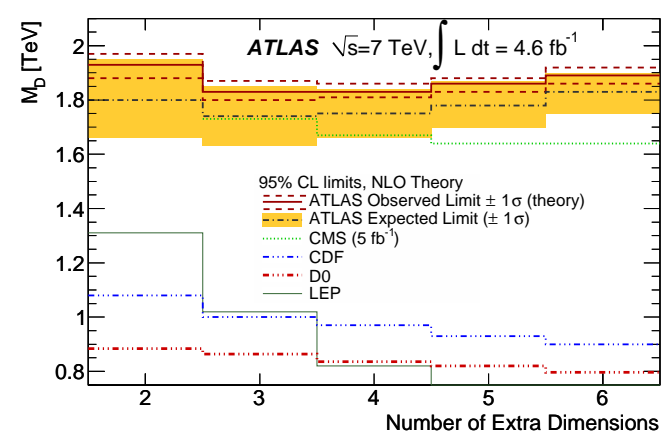

Figure 8: Observed (solid lines) and expected (dasheddotted lines) $95 \% \mathrm{CL}$ limits on $M_{D}$ as a function of the number of extra dimensions $n$ in the ADD LED model. The dashed lines around the observed limit indicate the impact of the $\pm 1 \sigma$ NLO theoretical uncertainty on the limit computation. The shaded band indicates the expected $\pm 1 \sigma$ range of limits in the absence of a signal [7].

not supersede the limits obtained with the $7 \mathrm{TeV}$ data due to the increase of the SM background and the lack of sufficient statistics in the MC samples employed to predict it.

\subsubsection{WIMP production using effective field theory}

The results of the monojet and monophoton analyses are interpreted in terms of WIMP dark matter production. The interaction is assumed to be a contact interaction and treated using an effective field theory approach with different operators with couplings characterized by a suppression scale $M_{*}$.

Systematic uncertainties on WIMP pair production are treated similarly to those of the ADD limits. The total 


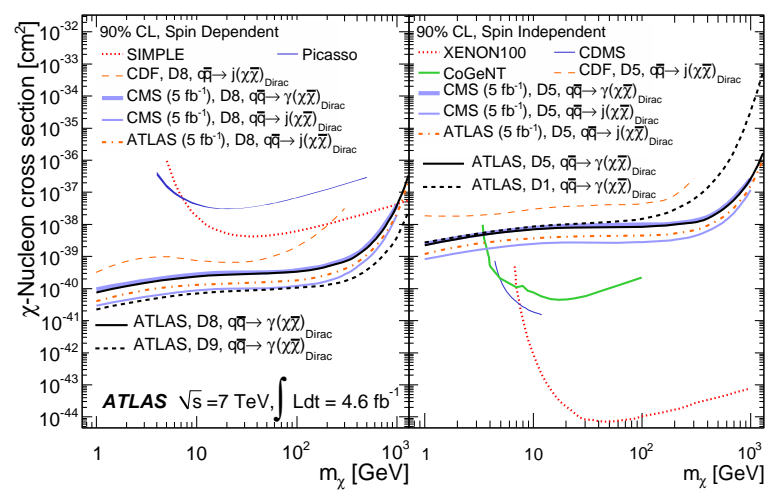

Figure 9: Monojet and monophoton 90\% CL upper limits on the nucleon-WIMP cross section as a function of $m_{\chi}$ for spin-dependent (left) and spin-independent (right) interactions for different operators. The results are compared with previous CDF and CMS results and results from direct WIMP detection experiments [6].

experimental uncertainty is dominated by jet and $E_{\mathrm{T}}^{\mathrm{miss}}$ energy scales and it is at the level of $10 \%$. The parton shower uncertainty is very relevant in this case because the signal production involves an ISR jet or photon. The PDF, factorisation and renormalisation scales uncertainties depend greatly on the WIMP mass and on the choice of the operator. More details are given in the original papers [5-7].

Figure 9 summarizes the $7 \mathrm{TeV}$ results of the monojet and monophoton [6, 7] for 90\% CL upper limits on the nucleon-WIMP cross section as a function of $m_{\chi}$ for spindependent and spin-independent operators. It includes also a comparison with the previous collider results and results from direct detection experiments [9-14]. The $8 \mathrm{TeV}$ monojet results [5] are slightly more restrictive than the $7 \mathrm{TeV}$ results for some of the operators. Assuming the validity of the effective field theory, the constraints put by colliders on the WIMP-nucleon cross section dominate for spin-dependent interactions over the full range of $m_{\chi}$, while they remain very restrictive for spin-independent interactions at low WIMP mass.

\subsubsection{Light gravitino production in GMSB}

This interpretation is done using the $8 \mathrm{TeV}$ monojet results reported in [5]. Based on SR3, limits at 95\% CL on the cross section are set for the associated production of gravitino and gluino/squark. The acceptance of this process depends on the masses of the squark and the gluino in the final state. In the degenerated mass case of squark and gluino $\left(m_{\tilde{g}}=m_{\tilde{q}}\right)$, the signal acceptance for the SR3 selection criteria is of the order of $30 \%$ for squark and gluino masses of about $1-2 \mathrm{TeV}$. The systematic uncertainties on the SUSY signal yields are determined in a similar way in the ADD and WIMP models. The experimental uncertainties are dominated by the jet and $E_{\mathrm{T}}^{\text {miss }}$ energy scales and resolutions are at the level of $15 \%$. Uncertainties due to the modeling of the parton shower, to PDFs and to renor-

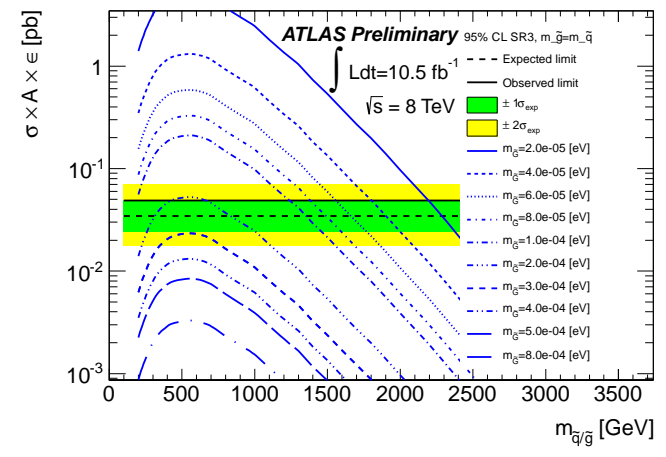

Figure 10: Cross section times acceptance times efficiency for the gravitino+squark/gluino production as a function of the squark/gluino mass in the case of degenerate squark and gluino. Different values for the gravitino mass are considered and the predictions are compared with modelindependent limits [5].

malization and factorization scales are determined using dedicated samples and their typical values are 10\%, 30\% and $35 \%$.

Figure 10 presents, for the case of degenerate squark and gluino, the cross section times acceptance times efficiency $(\sigma \times A \times \epsilon)$ as a function of the squark/gluino mass for different gravitino masses. For comparison, the modelindependent 95\% CL limits are shown. Expected and observed $95 \%$ CL limits on the gravitino-squark/gluino mass plane are presented in Figure 11. Gravitino masses below $1 \times 10^{-4} \mathrm{eV}\left(4 \times 10^{-5} \mathrm{eV}\right)$ are excluded at $95 \% \mathrm{CL}$ for squark/gluino masses of $500 \mathrm{GeV}(1.7 \mathrm{TeV})$. These results significantly improve previous results at LEP and the Tevatron and constitute the best bounds on the gravitino mass to date.

\section{Summary and conclusions}

We reported in this article the most recent ATLAS results on the search for new phenomena in events with an energetic jet or photon, and large missing transverse momentum.

The measurements are in agreement with the SM predictions for the background, allowing to set modelindependent $95 \%$ CL upper limits on the fiducial cross section of new physics processes. In addition, 95\% CL limits on $M_{D}$ versus the number of extra spatial dimensions, in the ADD LED model, and on the pair production of WIMP dark matter candidate. The results are also interpreted in terms of limits on the production of a light gravitino in association with gluino or scalar quarks in a gauge-mediated supersymmetric model, leading to the best lower bound to date on the gravitino mass.

\section{References}

[1] N. Arkani-Hamed, S. Dimopoulos and G.R. Dvali, Phys. Lett. B 429, 263 (1998). 
[2] J. Goodman et al., Phys. Rev. D 82, 116010 (2010).

[3] G. Giudice and R. Rattazzi, Phys. Rept. 322, 419-499 (1999).

P. Fayet, Phys. Lett. B 70, 461 (1977).

R. Casalbuoni, S. De Curtis, D. Dominici, F. Feruglio and R. Gatto, Phys. Lett. B 215, 313 (1988).

[4] ATLAS Collaboration, JINST 3, S08003 (2008).

[5] ATLAS Collaboration, ATLAS-CONF-2012-147, https://cds.cern.ch/record/1493486

[6] ATLAS Collaboration, JHEP 04, 075 (2013).

[7] ATLAS Collaboration, Phys. Rev. Lett. 110, 011802 (2013).

[8] M.L. Mangano et al., JHEP 07, 001 (2003).

[9] CDF Collaboration, Phys. Rev. Lett. 108, 211804 (2012).

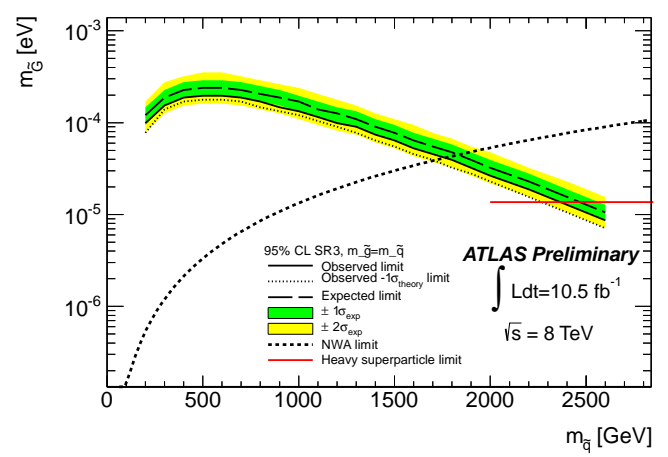

Figure 11: Observed (solid line) and expected (dashed line) $95 \% \mathrm{CL}$ lower limits on the gravitino mass as a function of the squark mass for degenerate squark/gluino masses. The dotted line indicates the impact on the observed limit of the $\pm 1 \sigma$ LO theoretical uncertainty. The shaded bands around the expected limit indicate the expected $\pm 1 \sigma$ and $\pm 2 \sigma$ ranges of limits in the absence of a signal. The dashed-dotted line defines the validity of the narrow-width approximation (see body of the text). The solid red line denotes the current limit from LEP [15] on the gravitino mass assuming very heavy squark/gluino [5].
[10] CMS Collaboration, Phys. Rev. Lett. 108, 261803 (2012).

[11] XENON100 Collaboration, Phys. Rev. Lett. 107, 131302 (2011).

[12] CDMS Collaboration, Phys. Rev. Lett. 106, 131302 (2011).

[13] M. Felizardo et al., Phys. Rev. Lett. 108, 201302 (2012).

[14] PICASSO Collaboration, Phys. Lett. B 711, 153 (2012).

[15] LEP2 SUSY Working Group Collaboration, Single Photons, 183-208 GeV, http://lepsusy.web.cern.ch/lepsusy/www/photons/single/ single_public_summer04.html 